\title{
Über das Vorkommen und die partielle Reinigung einer unspezifischen Cholinesterase in dem Eiweißdrüsensekret von Helix pomatia
}

\author{
Von R. Voigtmann und G. UhLENBRUCK \\ Aus der Medizinischen Universitätsklinile (Direktor: Prof. Dr. R. Gross), Köln-Lindenthal
}

(Eingegangen am 19. August 1971)

Es wird das Vorkommen und die partielle Reinigung einer unspezifischen Cholinesterase im Eiweißdrüsensekret der Schnecke Helix pomatia beschrieben. Die Reinigungsschritte nach Anionenaustauschchromatographie und Gel-Filtration werden mit einem Immunserum gegen das Eiweißdrüsensekret in der Immunelektrophorese verfolgt. Die Eigenschaften und die eventuelle funktionelle Bedeutung des Enzyms werden diskutiert.

\section{Occurrence and partial purification of an unspecific cholinesterase in the albumin gland extract of Helix pomatia}

The occurrence and the partial purification of an unspecific cholinesterase in the albumin gland extract of the snail Helix pomatia are described. Anion-exchange-chromatography and gelfiltration are used for purification and each step is checked by immunoelectrophoresis with an immunserum against the albumin gland extract. The properties and functional importance of this enzyme are discussed.

Das Vorkommen eines "Anti-A"-Agglutinins in dem Sekret der Eiweißdrüse von Helix pomatia ist schon länger bekannt (1). Kürzlich konnten wir in diesem Sekret vier verschiedene Proteaseinhibitoren nachweisen (2). Bei der Untersuchung der Frage, ob diese Inhibitoren auch gegen andere Hydrolasen aktiv sind, stießen wir in dem Eiweißdrüsensekret der Schnecke auf ein Cholinester-spaltendes Enzym. Die vorliegende Arbeit beschreibt die Darstellung und die partielle Reinigung dieses Enzyms.

\section{Material und Methoden \\ Eiveißdriisensekret}

- Nach Präparation der Eiweißdrüse wurde das Sekret durch Extraktion mit $0,145 \mathrm{M} \mathrm{NaCl}$-Lösung gewonnen. Ungelöste Anteile wurden durch Zentrifugieren für $15 \mathrm{Min}$. bei $30000 \mathrm{~g}$ entfernt. Vor der chromatographischen Trennung wurde das Sekret 48 Stdn. gegen $0,02 \mathrm{M}$ Phosphatpuffer $\mathrm{pH} 8,0$ bei $4^{\circ}$ dialysiert.

\section{Enzymbestimmung}

Als Substrat für das Enzym wurden 0,12M Acetylthiocholinjodid (Serva, Heidelberg) und 0,12M Butyrylthiocholinjodid (Merck, Darmstadt) in $0,02 \mathrm{M}$ Phosphatpuffer $\mathrm{pH} 7,8$ verwandt. Als Indikator diente $0,25 \mathrm{~mm} 5,5^{\prime}$-Dithiobis-(2-nitrobenzoesäure), reinst (Serva, Heidelberg) in 0,02M Phosphatpuffer $\mathrm{pH} 7,8$, die durch das enzymatisch freigesetzte Thiocholinjodid im molaren Verhältnis von 1:1 zum gelb gefärbten 5-Mercapto-2-nitrobenzoat reduziert wird (3). Pro Enzymbestimmung wurden $2 \mathrm{ml} 0,25 \mathrm{~mm}$ 5,5'-Dithiobis-(2-nitrobenzoesäure), $0,1 \mathrm{ml}$ Substrat-Lösung und $0,1 \mathrm{ml}$ Enzym-Lösung eingesetzt. Als Kontroll- und Referenzwert dienten $2,1 \mathrm{ml}$ Indikator-Lösung und' $0,1 \mathrm{ml}$ SubstratLösung. In einem Beckman DB-Spektralphotometer wurde bei einer Wellenlänge von $412 \mathrm{~nm}$ die Extinktionszunahme pro Minute gemessen. Als Maß für die Enzymmenge in 0,1 ml EnzymLösung diente der Substratumsatz/Min. in $\mu \mathrm{Mol}$. Bei einem Extinktionskoeffizienten $\varepsilon_{412}=136001 \cdot \mathrm{Mol}^{-1} \cdot \mathrm{cm}^{-1}$ (4) für das gebildete 5-Metcapto-2-nitrobenzoat entspricht eine Extinktionszunahme von $0,01 / \mathrm{Min}$. in dem gewählten Reaktionsvolumen von $2,2 \mathrm{ml}$ einem Umsatz von $1,62 \times 10^{-3} \mu \mathrm{Mol}$ Substrat pro Minute, entsprechend 1,62 mU. Unter stets gleichbleibenden Bedingungen ergibt die Multiplikation des gemessenen $\triangle \mathrm{E} /$ Min. mit dem Faktor 162 den Substratumsatz pro Minute in $\mathrm{nMol}$ durch $0,1 \mathrm{ml}$ Enzym-Lösung in einem Reaktionsansatz von $2,2 \mathrm{ml}$ entsprechend $\mathrm{mU} / 0,1 \mathrm{ml}$ Enzymlösung. Alle Enzymbestimmungen wurden bei $25^{\circ}$ ausgeführt.

\section{Anionenaustauscbcbromatographie}

DEAE-Cellulose Type DEAE-SS der Firma Serva (Heidelberg) wurde nacheinander mit $0,1 \mathrm{~N} \mathrm{NaOH}$, bidest. Wasser, $0,1 \mathrm{~N} \mathrm{HCl}$ und wiederum mit bidest. Wasser ausgewaschen. Nach Füllung der Säule $(30 \mathrm{~cm} \times 1,5 \mathrm{~cm})$ wurde der Anionenaustauscher mit $0,02 \mathrm{M}$ Phosphatpuffer $\mathrm{pH} \mathrm{8,0}$ äquilibriert, bis der $\mathrm{pH}$-Wert des Eluates den des eingesetzten Puffers erreicht hatte. Die Elution des Eiweißdrüsensekretes führten wir zunächst mit $0,02 \mathrm{M}$ Phosphatpuffer und darauf mit einem linear ansteigenden $\mathrm{NaCl}-\mathrm{Gra}$ dienten in dem gleichen Puffer durch. Die Flußrate betrug $18 \mathrm{ml} /$ Std. Sämtliche chromatographischen Trennungen wurden bei $5^{\circ}$ ausgeführt und das Eluat in $2 \mathrm{ml}$ Fraktionen in einem gekühlten Beckman Fraktionssammler gesammelt.

\section{Gel-Filtration}

Sephadex G 200 der Firma Pharmacia (Uppsala, Schweden) wurde durch fünfstündiges Erhitzen auf $105^{\circ}$ in bidest. Wasser zum Quellen gebracht. Das gequollene $\mathrm{Gel}$ wurde nach dem $\mathrm{Ab}$ kühlen mittels einer Saugflasche von Luft befreit und in eine Säule $(60 \mathrm{~cm} \times 1,5 \mathrm{~cm})$ gepackt. Nach Åquilibrierung der Säulenfüllung mit 0,02M Phosphatpuffer $\mathrm{pH} 7,8$ erfolgte die Elution mit dem gleichen Puffer und einer Flußrate von $16 \mathrm{ml} / \mathrm{Std}$.

\section{Immunelektrophorese}

Dic Immunelektrophorese wurde in 1proz. Agarose (Serva, Heidelberg) in Veronalpuffer vom pH-Wert 8,6 und der Ionenstärke 0,1 in einem LKB-Gerät durchgefühtt. Die Laufzeit der Elektrophorese betrug $45 \mathrm{Min}$. bei einem Spannungsabfall von $9 \mathrm{~V} / \mathrm{cm}$ und einer Stromstärke von $10,5 \mathrm{~mA}$. 20 Stdn. nach Einfüllen des Immunserums wurden die Präzipitate photographiert.

Das Immunserum vom Kaninchen gegen das Eiweißdrüsensekret wurde uns freundlicherweise von Herrn Prof. Dr. Ishiyama, Department of Legal Medicine, University Tokio, Tokio Japan, zur Verfügung gestellt.

\section{Analytische Methoden}

Der Eiweißgehalt der verschiedenen enzymenthaltenden Fraktionen wurde nach LowRY (5) mit hochgereinigtem Rinderalbumin als Standard bestimmt. 


\section{Hemmersucbe}

Prostigmin der Firma Hoffmann-La Roche AG (Grenzach) wurde als Inhibitor für das Enzym in einer Konzentration von $0,5 \mathrm{mg} / \mathrm{ml}$ eingesetzt.

Zum Nachweis der eventuellen Aktivitätshemmung des Enzyms durch das Immunserum inkubierten wir $0,2 \mathrm{ml}$ gereinigte EnzymLösung mit 0,2 $\mathrm{ml}$ Immunserum für $60 \mathrm{Min}$. bei Raumtemperatur und maßen die Enzymaktivität wie oben beschrieben. Kontrollen führten wir mit mit $0,145 \mathrm{M} \mathrm{NaCl}-\mathrm{Lösung} 1: 1$ verdünntem Immunserum und Enzym-Lösung durch.

\section{Ergebnisse}

Abbildung 1 zeigt den ersten chromatographischen Trennungsschritt von $3 \mathrm{ml}$ Eiweißdrüsensekret mit einem Eiweißgehalt von 3\% über DEAE-Cellulose.

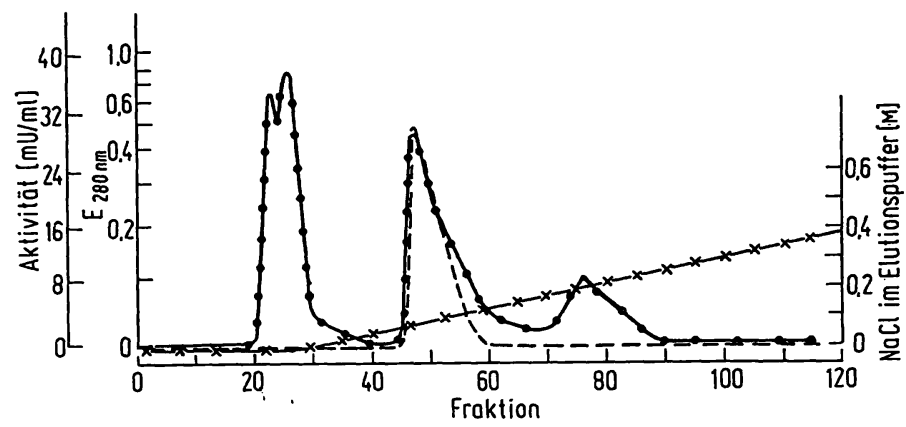

Abb. 1

Chromatogramm von $3 \mathrm{ml}$ Eiweißdrüsensekret mit einem Eiweißgehalt von $3 \%$ über eine DEAE-Cellulose Säule $(30 \mathrm{~cm} \times 1,5 \mathrm{~cm})$ Elution mit linear ansteigenden $\mathrm{NaCl}$-Gradienten in $0,02 \mathrm{M}$ Phosphatpuffer pH 8,0 $\times-x$,

Substratumsatz durch $0,1 \mathrm{ml}$ Eluat in 2,2 ml Reaktionsvolumen, angegeben in $\mathrm{mU} / \mathrm{ml}$ Eluat - -

Extinktion des Eluates bei $280 \mathrm{~nm} \cdot-\bullet$,

Flußrate $18 \mathrm{ml} / \mathrm{Std}$., Fraktionsvolumen $2 \mathrm{ml}$

Es lassen sich drei deutlich voneinander getrennte Komponenten unterscheiden, wobei die Fraktionen 20-35 nicht an den Anionenaustauscher gebunden werden und mit dem Phosphatpuffer allein eluiert werden können. Sie enthalten das gesamte „Anti-A“-Agglutinin des Sekretes und keine Enzymaktivität. Nur die Fraktionen $44-56$, die bei $0,05 \mathrm{M} \mathrm{NaCl}$ desorbiert werden, enthalten das Enzym. Die Fraktionen 70-90 zeigen weder „Anti-A“- noch Enzymaktivität.

Die enzymenthaltenden Fraktionen werden vereinigt, eingeengt auf etwa $4 \mathrm{ml}$ und $24 \mathrm{Stdn}$. bei $4^{\circ}$ gegen $0,02 \mathrm{M}$ Phosphatpuffer $\mathrm{pH} 7,8$ dialysiert.

Die vergleichende Immunelektrophorese des gesamten Eiweißdrüsensekretes (oben) und der konzentrierten Fraktionen 44-56 (unten) in Abbildung 2 verdeutlicht den ersten Reinigungsschritt des Enzyms.

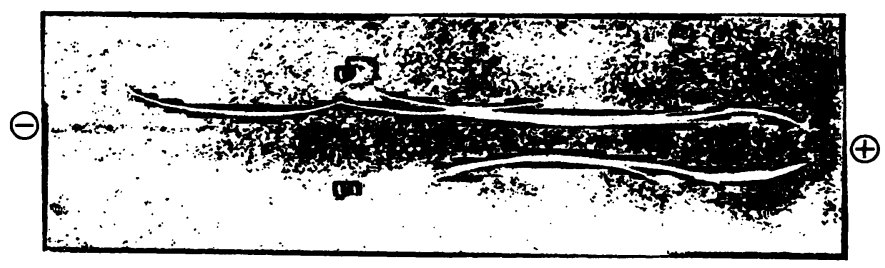

Abb. 2

Immunelektrophorese des Eiweißdrüsensekretes (oben) und der vereinigten, konzentrierten Fraktionen 44-56 (unten) in 1 proz. Agarose in Veronalpuffer mit einem pH von 8,6 und einer Ionenstärke von 0,1 , Laufzeit $45 \mathrm{Min}$. bei einem Spannungsabfall von $9 \mathrm{~V} / \mathrm{cm}$ und einer Stromstärke von $10,5 \mathrm{~mA}$, Präzipitate 20 Stdn. nach Einfüllen des Immunserums (Graben) photographiert
Während man bei dem Gesamtextrakt mindestens 11 Präzipitate unterscheiden kann, finden sich in den gereinigten Fraktionen 44-56 lediglich noch 5 Präzipitate, die alle auf der Anodenseite liegen.

Abbildung 3 zeigt die weitere Auftrennung von $3 \mathrm{ml}$ der konzentrierten, enzymenthaltenden Fraktion nach Anionenaustauschchromatographie mit einem Eiweißgehalt von $0,6 \%$ über Sephadex G, 200 .

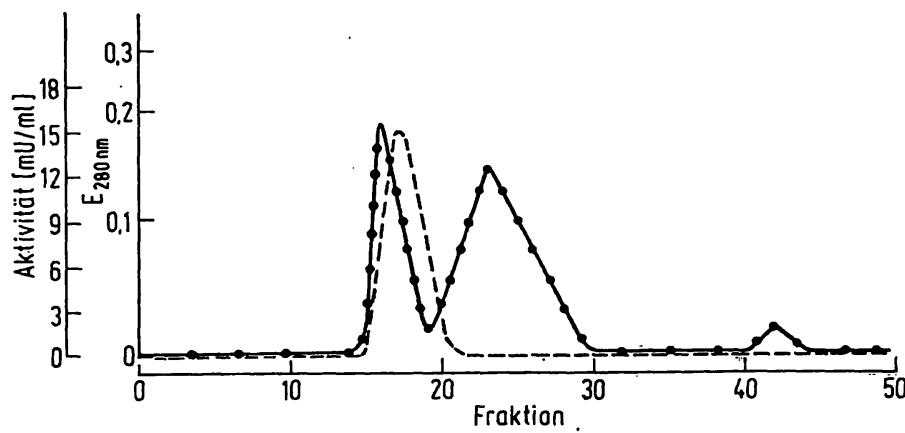

Abb. 3

Gel-Filtration über Sephadex G $200(60 \mathrm{~cm} \times 1,5 \mathrm{~cm})$ der vereinigten, konzentrierten Fraktionen 44-56 (3 ml) mit einem Eiweißgehalt von konzentrierten Fraktionen 44.56 (3 ml) mit einem Eiweisgehalt von durch $0,1 \mathrm{ml}$ Eluat in $2,2 \mathrm{ml}$ Reaktionsvolumen, angegeben in $\mathrm{mU} / \mathrm{ml}$ Eluat - - -

Extinktion des Eluates bei $280 \mathrm{~nm}$

Flußrate $16 \mathrm{ml} / \mathrm{Std}$., Fraktionsvolumen $2 \mathrm{ml}$

Auch hier trennen sich wiederum drei Komponenten, wobei das Enzym in den Fraktionen 17-20 gefunden wurde.

Abbildung 4 veranschaulicht in der Immunelektrophorese die weitere Reinigung des Enzyms. Während die konzentrierten Fraktionen 44-56 (oben) noch in 5 Banden mit dem Immunserum präzipitierten, sieht man in der Fraktion 18 nach Gel-Filtration nur noch 2 Präzipitationslinien.

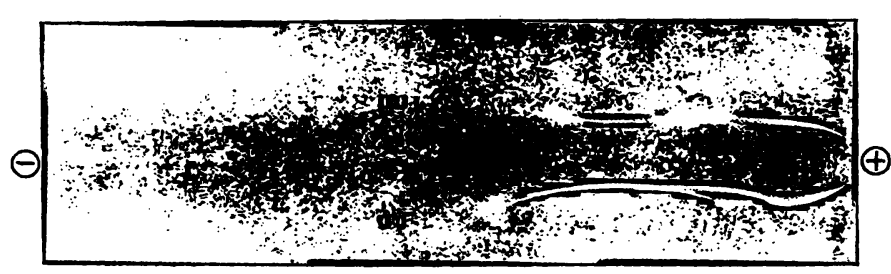

Abb. 4

Immunelektrophorese der Fraktion 18 nach Sephadex-Gel-Filtration (oben) und der konzentrierten Fraktionen 44-56 nach Anionenaustauschchromatographie (unten) in 1proz. Agarose in Veronalpuffer mit einem pH von 8,6 und einer lonenstärke von 0,1 , Laufzeit 45 Min. bei einem Spannungsabfall von $9 \mathrm{~V} / \mathrm{cm}$ und einer Stromstärke von $10,5 \mathrm{~mA}$, Präzipitate $20 \mathrm{Stdn}$. nach Einfüllen des Immunserums (Graben) photographiert

Die Tabelle 1 gibt eine zusammenfassende Darstellung über die Reinigung und Anre: nerung des Enzyms.

Insgesamt wurde etwa eine fünfzehnfache Anreicherung des Enzyms erreicht. Auch die Immunelektrophorese des Gesamtextraktes (oben) und der Fraktion 18 nach Gel-Filtration (unten) zeigt deutlich die weitgehende Reinigung der unspezifischen Cholinesterase an (Abb. 5). 
Tab. 1

Eiweißgehalt und Enzymmenge verschiedener Fraktionen bei der Reinigung einer unspezifischen Cholinesterase aus Helix pomatia

\begin{tabular}{|c|c|c|c|}
\hline$\cdot$ & $\begin{array}{l}\text { Eiweißgehalt } \\
{[\mathrm{mg} / \mathrm{ml}]}\end{array}$ & $\begin{array}{c}\text { Spezif. Aktivität } \\
{[\mathrm{mU} / \mathrm{mg}]}\end{array}$ & Anreicherung \\
\hline Eiweißdrüsensekret vor Auftrennung & 30 & 2,21 & \\
\hline $\begin{array}{l}\text { Konzentrierte Fraktionen } 44-56 \text { nach } \\
\text { lonenaustauschchromatographie }\end{array}$ & 6 & 11,06 & etwa 5 fach \\
\hline Fraktion 18 nach Gel-Filtration & 0,98 & 34,72 & etwa 15 fach \\
\hline
\end{tabular}

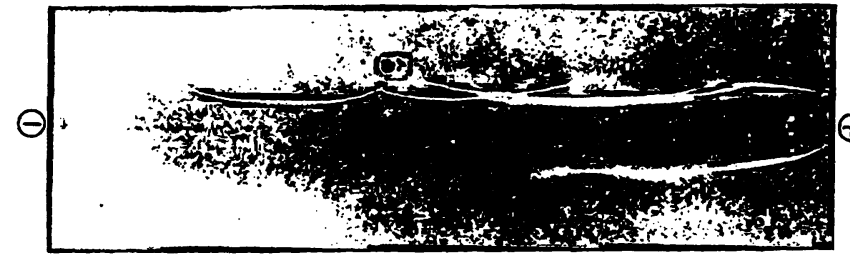

Abb. 5

Immunelektrophorese des Eiweißdrüsensekretes (oben) und der Fraktion 18 nach Gel-Filtration (unten) in 1 proz. Agarose in Veronalpuffer mit einem pH von 8,6 und einer lonenstärke von 0,1 , Laufzeit $45 \mathrm{Min}$. bei einem Spannungsabfall von $9 \mathrm{~V} / \mathrm{cm}$ und einer Stromstärke von 10,5 mA, Präzipitate 20 Stdn. nach Einfüllen des Immunserums (Graben) photographiert

Zur weiteren Charakterisierung untersuchten wir die Substratspezifität der gereinigten Enzympräparation. Das Enzym wies die gleiche Umsatzrate/Min. gegenüber Acetylthiocholin wie gegenüber Butyrylthiocholin auf. Die Aktivität in $0,1 \mathrm{ml}$ Enzym-Lösung betrug für Acetylthiocholinjodid $6,64 \mathrm{mU}$, die für Butyrylthiocholinjodid 6,48 mU.

Zur Einordnung des Enzyms in die Gruppe der Cholinesterasen untersuchten wir die Hemmung durch Prostigmin. Wir beobachteten eine vollkommene Hemmung bei einer eingesetzten Konzentration des Inhibitors von $0,25 \mathrm{mg} / \mathrm{ml}$.

Nach der Inkubation des gereinigten Enzyms mit dem Immunserum ließ sich kein Aktivitätsverlust feststellen. Während der Substratumsatz im Inkubationsgemisch $18,63 \mathrm{nMol} / \mathrm{Min}$. betrug, ergab die Summe beider Kontrollwerte 18,30 nMol/Min., die sich aus der Umsatzrate des verdünnten Immunserums von 14,90 nMol/Min. und dem des verdünnten Enzyms von 3,40 nMol/Min. zusammensetzt. Weiterhin wurde die Temperaturempfindlichkeit des Enzyms untersucht. Durch halbstündiges Erwärmen auf $58^{\circ}$ wurde die Aktivität um $70 \%$ reduziert.

\section{Diskussion}

Während sich in der Vergangenheit das Augenmerk vieler Forschergruppen nur auf das Vorkommen und die Isolierung des „Anti-A“-Agglutinins im Eiweißdrüsensekret von Schnecken $(1,6)$ richtete, konnten wir erstmals anknüpfend an die Arbeit von UHLENBRUCK und Mitarbeitern (2), die das Vorkommen von vier verschiedenen Proteaseinhibitoren im Eiweißdrüsensekret beschrieben, ein Cholinester-spaltendes Enzym in diesem Sekret nachweisen. Wie Abbildungen 1 und 3 zeigen, gelang uns über die Anionenaustauschchromatographie und dic Gel-Filtration eine weitgehende Reinigung des Enzyms, was durch die immunelektrophoretischen
Untersuchungen in den Abbildungen 2, 4 und 5 untermauert wird. Die gereinigte Enzympräparation präzipitiert mit dem Immunserum in einer zur Anode gewanderten Bande und in einer zweiten, das Enzym enthaltenden Bande. Dieser Befund von zwei Präzipitationslinien deutete sich auch schon in der Gel-Filtration über Sephadex G 200 an. Wie aus Abbildung 3 hervorgeht, liegt die höchste Enzymkonzentration des untersuchten Eluates leicht hinter dem Maximum der Extinktion bei $280 \mathrm{~nm}$. In den aktiven Fraktionen 17, 18 und 19 fällt die Extinktion bei $280 \mathrm{~nm}$ schon deutlich ab. Die fehlende Retention des Enzyms im Sephadex-Gel läßt ein Molekulargewicht von über 200000 vermuten.

Aus der Relation des Eiweißgehaltes der verschiedenen Fraktionen zum Enzymgehalt ergibt sich, wie Tabelle 1 zeigt, eine 15 fache Anreicherung.

Das Enzym wird durch Prostigmin vollkommen gehemmt; es ist daher in die Gruppe der Cholinesterasen einzuordnen. Da Prostigmin ein sehr spezifischer Inhibitor für Cholinesterasen ist, konnte die Möglichkeit einer unspezifischen Esteraseaktivität auf die angebotenen Substrate ausgeschlossen werden. Die weitere Klassifizierung des Enzyms erfolgte durch die Aktivitätsbestimmung gegenüber verschiedenen Substraten. Nach Augustrnsson (7) lassen sich die spezifische oder wahre Acetylcholinesterase und die unspezifische oder Pseudo-Cholinesterase unter anderem durch ihr unterschiedliches Verhalten gegenüber Butyrylthiocholinjodid differenzieren. Während die unspezifische Cholinesterase sowohl den Acetyl- als auch den Butyrylester des Thiocholinjodids zu spalten vermag, hydrolysiert die spezifische Acetylcholinesterase nur das Acetylthiocholinjodid. Die Umsatzraten betrugen unter den angegebenen Testbedingungen für das von uns gereinigte Enzym 6,64 bzw. 6,48 nMol/Min. Danach ist das Enzym in die Gruppe der Pseudocholinesterasen einzubeziehen.

Erwähnenswert scheint uns noch die zeit- und konzentrationsabhängige Inaktivierung des Enzyms in Tris$\mathrm{HCl}-\mathrm{Puffer} z \mathrm{u}$ sein. Schon bei der vorbereitenden Dialyse des Eiweißdrüsensekretes gegen $0,02 \mathrm{M}$ Tris- $\mathrm{HCl}$ Puffer $\mathrm{pH} 8,2$ über $48 \mathrm{Stdn}$. bei $4^{\circ}$ konnten wir einen Aktivitätsverlust von über $50 \%$, berechnet auf den Eiweißgehalt, gegenüber dem nicht dialysierten Sekret registrieren. Mit ansteigender Ionenstärke des Puffers, wie wir ihn bei einem Experiment zur Elution benutzten, wird das Enzym vollkommen inaktiviert. In 0,3 M TrisHCl-Puffer pH 8,2 konnten wir keine Enzymaktivität mehr nachweisen. $\mathrm{Ob}$ es sich hier ähnlich wie beim 
Prostigmin um eine Blockierung des Enzyms handelt, indem sich das N-Atom von Tris dem einen Reaktionszentrum der Cholinesterase anlagert, oder ob die Inaktivierung auf einer Denaturierung des Enzyms beruht, kann nicht schlüssig beantwortet werden. Der Befund jedoch, daß eine Reaktivierung des Enzyms nicht zu beobachten ist, wenn man es fünf Tage in der Kälte gegen 0,02M Phosphatpuffer dialysieren läßt, spricht eher für eine Denaturierung.

Die fehlende Inaktivierung des Enzyms nach Inkubation mit dem Immunserum macht - da das Enzym in der Immunelektrophorese präzipitiert wird - die unterschiedliche Lokalisation des aktiven Zentrums und der determinanten Gruppe am Proteinmolekül deutlich, wie dies auch für andere Enzyme schon beschrieben worden ist (8).
Uber die Funktion dieser unspezifischen Cholinesterase im Eiweißdrüsensekret von Schnecken lassen sich bis heute nur Vermutungen anstellen. Immerhin weist der Befund, daß dieses Enzym im Ei selbst in 3-4fach höherer Konzentration von uns gefunden wurde, darauf hin, daß die Eiweißdrüse, ähnlich wie es schon für das "Anti-A"-Agglutinin und die Proteaseinhibitoren beschrieben wurde (2), Substanzen produziert, die dem Ei mitgegeben werden. Ob es sich bei diesem Enzym auch um einen möglichen Schutz- und Abwehrmechanismus handelt, wie es für die Proteaseinhibitoren und auch für das „Anti-A“-Agglutinin diskutiert worden ist, muß noch unbeantwortet bleiben.

Die Untersuchungen wurden durch Mittel der Deutschen Forschungsgemeinschaft ermöglicht. Unser besonderer Dank gilt Frau Elke Jörges für die gute Assistenz bei dieser Arbeit.

\section{Literatur}

1. Prokop, O., Klin. Wschr. 47, 605 (1969). - 2. Uhlenbruck, G., I. SPRENGer und I. IshIYAMA, diese Z. 9, 361 (1971). - 3. ElLMAN, G. L., Arch. Biochem. Biophysics 74, 443 (1958). - 4. ElLmaN, G. L., Arch. Biochem. Biophysics 82, 70 (1959). - 5. Lowry, O. H., N. J. Rosebrough, A. L. FARR und R. J. RANDall, J. biol. Chemistry 193, 265 (1951). - 6. SCHNITZLER, S., W. KRÜGER,
D. Felix, H. David, I. Uerlings, M. Bötrger und W. Kuhn, diese Z. 9, 304 (1971). - 7. Augustinsson, K. B., Acta physiol. scand. 15, Suppl. 52 (1948). - 8. Cinader, B., Antibodies to Biologically Active Enzymes. Proc. 2nd Meeting of the Federation of European Biochemical Societies, Vienna, 1965, Vol. 1.

Prof. Dr. G. Uhlenbruck 5000 Köln 41

Kerpenerstr. 15 